\title{
Subspace Identification of Hammerstein Model with Unified Discontinuous Nonlinearity
}

\author{
Borhen Aissaoui, ${ }^{1,2}$ Moêz Soltani, ${ }^{1,2}$ and Abdelkader Chaari ${ }^{1,2}$ \\ ${ }^{1}$ National Higher Engineering School of Tunis (ENSIT), University of Tunis, 5 Av. Taha Husein, BP 56, 1008 Tunis, Tunisia \\ ${ }^{2}$ Laboratoire d'Ingenierie des Systèmes Industriels et des Energies Renouvelables (LISIER), University of Tunis, ENSIT, Tunis, Tunisia
}

Correspondence should be addressed to Borhen Aissaoui; borhen.issaoui@yahoo.fr

Received 10 June 2016; Revised 8 October 2016; Accepted 31 October 2016

Academic Editor: Qingsong Xu

Copyright (C) 2016 Borhen Aissaoui et al. This is an open access article distributed under the Creative Commons Attribution License, which permits unrestricted use, distribution, and reproduction in any medium, provided the original work is properly cited.

\begin{abstract}
The main aim of this study is to handle the case where the structures of nonlinear systems are unknown. In the many works, the parametric identification of nonlinear systems represented by Hammerstein model, with discontinuous and asymmetric nonlinearity, considers the structures of the nonlinear and linear blocks are known, especially the nonlinear bloc. To solve this problem, a unified form of nonlinearity representing eight cases of nonlinearities can be used. The parameters of both blocks, linear and nonlinear, are estimated using an iterative subspace approach. More importantly, in an attempt to show the extent to which this method is efficient, we apply it to experimental data obtained from the electropneumatic system. As a result, the numerical and experimental examples confirm a good conditioning and computational efficiency.
\end{abstract}

\section{Introduction}

Many physical and biological systems have been modelled as a Hammerstein structure, such as the Stretch Reflex at the human ankle [1], the mechanical behaviour of lung tissue [2] and the electropneumatic system. In the Hammerstein model, there are two cascaded linear and nonlinear blocks which are an interconnection between the static structure nonlinearity and the Linear Time-Invariant (LTI) dynamic subsystem. Indeed, a variety of methods have been developed to identify Hammerstein systems. Narendra and Gallman [3] developed an iterative identification algorithm. They separate the parameters into two sets: one corresponding to the nonlinear component and the second from the linear element. In addition, the cross correlation-based methods are applied in order to estimate the linear dynamic subsystem [4] where the algorithm updates the nonlinearity's output estimation at each step using the inverse dynamic of the linear component. Finally, they estimate and predict the inverse dynamics and identify the output nonlinearity. In other studies, the structures of the nonlinear and linear blocks are known in the case of continuous nonlinearity [5], discontinuous nonlinearities (DN) such as saturation with hysteresis (SH) [6], hysteresis-backlash [7], and hysteresisrelay nonlinearities [8].

In the real world, the identification of static nonlinearity subsystem remains the major problem in the Hammerstein model. Indeed, the structures of the nonlinear block are unknown and their estimate is important. Rejeb et al. [9] used the least squares method to estimate the parameters of unknown nonlinear block. Later, the same authors [10] proposed a general model combining discontinuous and asymmetric nonlinearities which generates all the possible combinations of the nonlinearities discontinuous elements. In some context, Vörös [11] developed a system with the general backlash which is based on appropriate switching and internal functions.

The identification using parametric approaches requires a minimal information priority about the system structure, that is, system order, noise model, and so forth [12]. Then, the LTI state model cannot be estimated through input-output measurements. Hence, the subspace method is introduced in order to overcome this problem. The subspace methods estimate the state space models for linear system with no 


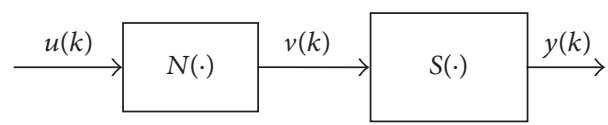

Figure 1: A discrete-time Hammerstein model.

a priori knowledge about the system [13]. In other words, those approaches are characterized by the simplicity of implementation including its effective results. In many cases, these methods provide a good alternative to the classical nonlinear optimization-based prediction-error methods [14].

Subspace identification is quite a well-accepted method for the identification of multivariable LTI systems. This method estimates an LTI state space model directly from input-output measurements. Moreover, Verhaegen and Varga [15] as well as Verhaegen and Westwick [16] proposed the Multivariable Output Error State Space identification model (MOESP) algorithm to identify Hammerstein models. In [1, 17, 18], the authors have treated the case of Single Input Single Output (SISO) Hammerstein model. Other authors proposed some subspace methods adapted to the HammersteinWienner $[19,20]$. Moreover, many researchers have developed a new identification subspace-based algorithm for the linear model [21-23].

Nevertheless, the structure of nonlinear block is used as known in those methods. Indeed, we developed an identification algorithm for a SISO Hammerstein model with discontinuous nonlinearities such as hysteresis, saturation, preload, and dead-zone. The proposed method uses an iterative subspace algorithm. The determination of the structure of nonlinearity is obtained based on Unified Discontinuous Nonlinearity (UDN) method. The parameters of both blocks are estimated simultaneous using the proposed iterative subspace approach.

This paper is organized as follows. Section 2 presents the problem formulation. Section 3 describes the proposed method. A numerical simulation validates the proposed algorithm and then applying to estimate the linear and nonlinear blocks model of electropneumatic system are shown in Section 4. Finally, this paper will be concluded in Section 5 .

\section{Problem Formulation}

Figure 1 presents a discrete-time SISO Hammerstein system, which is described by the following forms:

$$
S:\left\{\begin{array}{l}
x(k+1)=A x(k)+B v(k)+\omega(k), \\
y(k)=C x(k)+D v(k)+\varepsilon(k),
\end{array}\right.
$$

where $y(k) \in \mathbb{R}, x(k) \in \mathbb{R}^{n}, v(k) \in \mathbb{R}, \omega(k) \in \mathbb{R}^{n}$, and $\varepsilon(k) \in \mathbb{R}$ are the LTI system output, state, output of the nonlinearity, process noise, and output measurement noise vectors at discrete time $k$. $A \in \mathbb{R}^{n \times n}, B \in \mathbb{R}^{n \times 1}, C \in \mathbb{R}^{1 \times n}$, and $D \in \mathbb{R}$ are the unknown system matrices of the state space.

The discontinuous and asymmetric nonlinearity $N[\cdot]$ can be characterized by a linear function as

$$
v(k)=N(u(k))=g^{T} \varphi_{u}(k),
$$

TABLE 1: Different generated nonlinearities.

\begin{tabular}{lc}
\hline $\mathrm{DN}$ & Choices of parameters \\
\hline $\mathrm{UDN}$ & $p \neq 0, a \neq 0, b \neq 0, Z_{1} \neq 0, Z_{2} \neq 0, e_{1} \neq 0, e_{2} \neq 0$ \\
$\mathrm{DN}_{1}$ & $p \neq 0, a=b, b \neq 0, Z_{1} \neq 0, Z_{2} \neq 0, e_{1} \neq 0, e_{2} \neq 0$ \\
$\mathrm{DN}_{2}$ & $p \neq 0, a=b, b \neq 0, Z_{1} \neq 0, Z_{2} \neq 0, e_{1}=0, e_{2}=0$ \\
$\mathrm{DN}_{3}$ & $p \neq 0, a \neq 0, b \neq 0, Z_{1} \neq 0, Z_{2} \neq 0, e_{1}=0, e_{2}=0$ \\
$\mathrm{DN}_{4}$ & $p \neq 0, a \neq 0, b=0, Z_{1} \neq 0, Z_{2} \neq 0, e_{1}=0, e_{2}=0$ \\
$\mathrm{DN}_{5}$ & $p \neq 0, a=0, b \neq 0, Z_{1} \neq 0, Z_{2} \neq 0, e_{1} \neq 0, e_{2} \neq 0$ \\
$\mathrm{DN}_{6}$ & $p \neq 0, a=0, b=0, Z_{1} \neq 0, Z_{2} \neq 0, e_{1} \neq 0, e_{2} \neq 0$ \\
$\mathrm{DN}_{7}$ & $p \neq 0, a=0, b \neq 0, Z_{1} \neq 0, Z_{2} \neq 0, e_{1}=0, e_{2}=0$ \\
$\mathrm{DN}_{8}$ & $p \neq 0, a=0, b=0, Z_{1} \neq 0, Z_{2} \neq 0, e_{1}=0, e_{2}=0$ \\
\hline
\end{tabular}

with

$$
\begin{aligned}
g & =\left[g_{1}, \ldots, g_{r}\right]^{T}, \\
\varphi_{u}(k) & =\left[\varphi_{1}(u(k)), \ldots, \varphi_{r}(u(k))\right]^{T},
\end{aligned}
$$

where $g \in \mathbb{R}^{r \times 1}$ is the unknown nonlinearity parameter vector and $\varphi_{u}(k) \in \mathbb{R}^{r \times 1}$ is the observation vector.

In our work, the process noise $\omega(k)$ is considered zero. Then, replacing (2) in (1), we obtain

$$
S:\left\{\begin{array}{l}
x(k+1)=A x(k)+B g^{T} \varphi_{u}(k), \\
y(k)=C x(k)+D g^{T} \varphi_{u}(k)+\varepsilon(k) .
\end{array}\right.
$$

The identification problem is how to estimate the nonlinear parameter vector $g$, the dynamic matrices $(A, B, C, D)$, and the model order $n$ from input-output data set.

\section{Methods}

In this section, we present a subspace identification algorithm for a SISO Hammerstein model with Unified Discontinuous Nonlinearities.

3.1. Unified Discontinuous Nonlinearity. Figure 2 illustrates the $v(k)$ evolution with respect to the input $u(k)$ of the nonlinear block using UDN [9]. This latter contains an asymmetric piecewise function with a hysteresis, a saturation, a preload, a dead-zone, and so forth.

The UDN is characterized by the unknown parameters $p, a, b, Z_{1}, Z_{2}, e_{1}$, and $e_{2} . Z_{1}$ and $Z_{2}$ are scalar positive parameters that characterize the saturation, $a \geq 0$ and $b \geq 0$ define the width and the centre of the hysteresis as well as the dead-zone width, $p$ is the hysteresis slope. $e_{1}$ and $e_{2}$ define the preload thresholds. The different choices of these parameters lead to the definition of eight nonlinearities which are listed in Table 1 and shown in Figure 3.

Using Table 1, the general form of $v(k)$ with respect to $u(k)$ and $\Delta u(k)$ for the different cases of nonlinearities can be defined as 


$$
v(k)= \begin{cases}p Z_{1}+e_{1} & \text { if } u(k)>Z_{1}+b+a, \Delta u(k)<0 \\ & \text { or } Z_{1}+b-a<u(k)<Z_{1}+b+a, \Delta u(k)<0 \\ p(u(k)-a-b)+e_{1} & \text { if } b+a \leq u(k) \leq Z_{1}+b+a, \Delta u(k)>0 \\ p(u(k)-a+b)+e_{2} & \text { if } Z_{2}-b+a \leq u(k) \leq-b+a, \Delta u(k)>0 \\ p(u(k)+a-b)+e_{1} & \text { if } b-a \leq u(k) \leq Z_{1}+b-a, \Delta u(k)<0 \\ p(u(k)+a+b)+e_{2} & \text { if } Z_{2}-b-a \leq u(k) \leq-b-a, \Delta u(k)<0 \\ p Z_{2}+e_{2} & \text { if } u(k)<Z_{2}-b-a, \Delta u(k)<0 \\ & \text { or } Z_{2}-b<u(k)<Z_{2}-b+a, \Delta u(k)>0 \\ e_{1} & \text { if } b-a<u(k)<b+a, \Delta u(k)>0 \\ e_{1} & \text { if }-b-a<u(k)<-b+a, \Delta u(k)<0 \\ 0 & \text { if }-b+a \leq u(k) \leq b-a \forall \Delta u(k),\end{cases}
$$

with $\alpha=h[\Delta u(k)]$ and $\Delta u(k)=u(k)-u(k-1)$.

Using (2), (5) can be rewritten as

$$
\begin{aligned}
v(k)= & p u(k) f_{1}(k)-p a f_{2}(k)+p b f_{3}(k)+p Z_{1} h_{1}(k) \\
& +p Z_{2} h_{2}(k)+e_{1} h_{1 \mathrm{pr}}(k)+e_{2} h_{2 \mathrm{pr}}(k) \\
= & g_{\mathrm{UDN}}^{T} \varphi_{\mathrm{UDN}}(k),
\end{aligned}
$$

with

$$
\begin{aligned}
f_{1} & (k)=\alpha\left[\left(1-\left(h\left[u(k)-\left(Z_{1}+b+a\right)\right]\right.\right.\right. \\
+ & h[(b+a)-u(k)]))+(1 \\
- & \left.\left.\left(h\left[\left(Z_{2}-b+a\right)-u(k)\right]+h[u(k)-(-b+a)]\right)\right)\right] \\
+ & (1-\alpha)\left[\left(1-\left(h\left[u(k)-\left(Z_{1}+b-a\right)\right]\right.\right.\right. \\
+ & h[(b-a)-u(k)]))+(1 \\
& -\left(h\left[\left(Z_{2}-b-a\right)-u(k)\right]\right. \\
& +h[u(k)-(-b-a)]))], \\
f_{2} & (k)=\alpha\left[1-\left(h\left[u(k)-\left(Z_{1}+b+a\right)\right]+h[(b+a)\right.\right. \\
& -u(k)])+\left(1-\left(h\left[\left(Z_{2}-b+a\right)-u(k)\right]\right.\right. \\
& +h[u(k)-(-b+a)]))]-(1-\alpha)[1-(h[u(k) \\
& \left.\left.-\left(Z_{1}+b-a\right)\right]+h[(b-a)-u(k)]\right)+(1 \\
& -\left(h\left[\left(Z_{2}-b-a\right)-u(k)\right]\right. \\
+ & h[u(k)-(-b-a)]))], \\
f_{3} & (k)=\alpha\left[\left(1-\left(h\left[u(k)-\left(Z_{1}+b+a\right)\right]\right.\right.\right. \\
+ & h[(b+a)-u(k)]))-(1 \\
- & \left.\left.\left(h\left[\left(Z_{2}-b+a\right)-u(k)\right]+h[u(k)-(-b+a)]\right)\right)\right]
\end{aligned}
$$

$$
\begin{aligned}
& +(1-\alpha)\left[\left(1-\left(h\left[u(k)-\left(Z_{1}+b-a\right)\right]\right.\right.\right. \\
& +h[(b-a)-u(k)]))-(1 \\
& -\left(h\left[\left(Z_{2}-b-a\right)-u(k)\right]\right. \\
& +h[u(k)-(-b-a)]))], \\
& h_{1}(k)=\alpha h\left[u(k)-\left(Z_{1}+b+a\right)\right]+(1-\alpha) h[u(k) \\
& \left.-\left(Z_{1}+b-a\right)\right], \\
& h_{2}(k)=\alpha h\left[\left(Z_{2}-b+a\right)-u(k)\right]+(1-\alpha) h\left[\left(Z_{2}-b\right.\right. \\
& -a)-u(k)], \\
& h_{1 \mathrm{pr}}(k)=h[u(k)-(b-a)], \\
& h_{2 \mathrm{pr}}(k)=h[(-b+a)-u(k)], \\
& h[\beta]= \begin{cases}1 & \text { if } \beta>0 \\
0 & \text { if } \beta \leq 0,\end{cases}
\end{aligned}
$$

where $h[\beta]$ is a switching sequence.

Using (6), (3) are rewritten as

$$
\begin{aligned}
& g_{\mathrm{UDN}}^{T}=\left[p, p a, p b, p Z_{1}, p Z_{2}, e_{1}, e_{2}\right] \\
& \varphi_{\mathrm{UDN}}(k)=\left[u(k) f_{1}(k),\right. \\
& \left.\quad-f_{2}(k), f_{3}(k), h_{1}(k), h_{2}(k), h_{1 \mathrm{pr}}(k), h_{2 \mathrm{pr}}(k)\right]^{T} .
\end{aligned}
$$

3.2. SH Nonlinearity. The nonlinearity output $v(k)$, represented by Figure $3\left(\mathrm{DN}_{4}\right)$, can be developed in piecewise as follows: 


$$
v(k)= \begin{cases}p Z_{1} & \text { if } u(k)>Z_{1}+a \text { or } Z_{1}-a<u(k)<Z_{1}+a, \Delta u(k)<0 \\ p(u(k)-a) & \text { if } Z_{2}+a \leq u(k) \leq Z_{1}+a, \Delta u(k)>0 \\ p(u(k)+a) & \text { if } Z_{2}-a \leq u(k) \leq Z_{1}-a, \Delta u(k)<0 \\ p Z_{2} & \text { if } u(k)<Z_{2}-a \text { or } Z_{2}-a<u(k)<Z_{2}+a, \Delta u(k)>0 .\end{cases}
$$

The set of the above piecewise defining $v(k)$ can be summarized in one equation as

$$
\begin{aligned}
v(k)= & p u(k) f_{1}(k)-p a f_{2}(k)+p Z_{1} f_{3}(k) \\
& +p Z_{2} f_{4}(k)
\end{aligned}
$$

with

$$
\begin{aligned}
& f_{1}(k)=\alpha[1 \\
& \left.-\left(h\left[u(k)-\left(Z_{1}+a\right)\right]+h\left[\left(Z_{2}+a\right)-u(k)\right]\right)\right] \\
& +(1-\alpha)[1 \\
& \left.\quad-\left(h\left[u(k)-\left(Z_{1}-a\right)\right]+h\left[\left(Z_{2}-a\right)-u(k)\right]\right)\right], \\
& f_{2}(k)=\alpha[1 \\
& \left.\quad-\left(h\left[u(k)-\left(Z_{1}+a\right)\right]+h\left[\left(Z_{2}+a\right)-u(k)\right]\right)\right] \\
& \quad-(1-\alpha)[1 \\
& \left.\quad-\left(h\left[u(k)-\left(Z_{1}-a\right)\right]+h\left[\left(Z_{2}-a\right)-u(k)\right]\right)\right], \\
& f_{3}(k)=\alpha h\left[u(k)-\left(Z_{1}+a\right)\right]+(1-\alpha) h[u(k) \\
& \left.\quad-\left(Z_{1}-a\right)\right], \\
& f_{4}(k)=\alpha h\left[\left(Z_{2}+a\right)-u(k)\right]+(1-\alpha) h\left[\left(Z_{2}-a\right)\right. \\
& -u(k)] .
\end{aligned}
$$

The parameter and observation vectors can be defined, respectively, as follows:

$$
\begin{aligned}
g_{\mathrm{SH}}^{T} & =\left[p, p a, p Z_{1}, p Z_{2}\right], \\
\varphi_{\mathrm{SH}}(k) & =\left[u(k) f_{1}(k), f_{2}(k), f_{3}(k), f_{4}(k)\right]^{T} .
\end{aligned}
$$

3.3. The Proposed Algorithm. In this section, we propose an iterative subspace identification algorithm in order to find the unknown parameters of UDN, order of the system, and the system matrices $(A, B, C, D)$.

Substituting (8) in (4), we obtain

$$
\begin{aligned}
x(k+1) & =A x(k)+\widetilde{B} \varphi_{\mathrm{UDN}}(k), \\
y(k) & =C x(k)+\widetilde{D} \varphi_{\mathrm{UDN}}(k)+\varepsilon(k),
\end{aligned}
$$

where $\widetilde{B}=\left[B g_{1 \mathrm{UDN}}, B g_{2 \mathrm{UDN}}, \ldots, B g_{r \mathrm{UDN}}\right]$ and $\widetilde{D}=$ $\left[D g_{1 \text { UDN }}, D g_{2}\right.$ UDN $, \ldots, D g_{r}$ UDN $]$ can be perceived as the LTI system state space realization. We can use MOESP algorithm
[24] to estimate the order of the linear part and the component of state space matrices $\widehat{A}, \widehat{\widetilde{B}}, \widehat{C}$, and $\widehat{\widetilde{D}}$ from input-output data.

The proposed algorithm minimizes the following cost function:

$$
\left(\widehat{B}, \widehat{D}, \widehat{g}_{\mathrm{UDN}}\right)=\underset{B, D, g_{\mathrm{UDN}}}{\arg \min } \quad\left\{\left\|\widehat{\widetilde{\Theta}}_{B D}-\widetilde{\Theta}_{B D}\right\|_{2}^{2}\right\},
$$

with

$$
\widetilde{\Theta}_{B D}=\left[\begin{array}{c}
B g_{1 \mathrm{UDN}}, \ldots, B g_{r \mathrm{UDN}} \\
D g_{1 \mathrm{UDN}}, \ldots, D g_{r \mathrm{UDN}}
\end{array}\right]
$$

The solution to this optimization problem (14) can be resolved using the Singular Value Decomposition (SVD) [25] of the matrix $\widehat{\Theta}_{B D}$ as

$$
\widehat{\widetilde{\Theta}}_{B D}=\left[\begin{array}{ll}
U_{1} & U_{2}
\end{array}\right]\left[\begin{array}{cc}
\Sigma_{1} & 0 \\
0 & \Sigma_{2}
\end{array}\right]\left[\begin{array}{c}
V_{1}^{T} \\
V_{2}^{T}
\end{array}\right],
$$

where $\Sigma_{1}=\operatorname{diag}\left\{\sigma_{1}, \ldots, \sigma_{r}\right\}, U_{1} \in \mathbb{R}^{n \times 1}$, and $V_{1} \in \mathbb{R}^{r \times 1}$.

The parameters of the different blocks are estimated iteratively using the proposed algorithm. Then, (8) are rewritten as follows:

$$
\begin{aligned}
& { }^{(s)} g_{\mathrm{UDN}}^{T}=\left[{ }^{(s)} \widehat{p},{ }^{(s)} \widehat{p}^{(s)} \widehat{a},{ }^{(s)} \widehat{p}^{(s)} \widehat{b},{ }^{(s)} \widehat{p}^{(s)} \widehat{Z}_{1},{ }^{(s)} \widehat{p}^{(s)} \widehat{Z}_{2},\right. \\
& \left.{ }^{(s)} \widehat{e}_{1},{ }^{(s)} \widehat{e}_{2}\right] \\
& { }^{(s)} \widehat{\varphi}_{\mathrm{UDN}}(k)=\left[u(k){ }^{(s)} \widehat{f}_{1}(k),-{ }^{(s)} \widehat{f}_{2}(k),{ }^{(s)} \widehat{f}_{3}(k),\right. \\
& \left.{ }^{(s)} \widehat{h}_{1}(k),{ }^{(s)} \widehat{h}_{2}(k),{ }^{(s)} \widehat{h}_{1 \mathrm{pr}}(k),{ }^{(s)} \widehat{h}_{2 \mathrm{pr}}(k)\right]^{T},
\end{aligned}
$$

with

$$
\begin{aligned}
& { }^{(s)} \widehat{f}_{1}(k)=\alpha\left[\left(1-\left(h\left[u(k)-\left({ }^{(s)} \widehat{Z}_{1}+{ }^{(s)} \widehat{b}+{ }^{(s)} \widehat{a}\right)\right]\right.\right.\right. \\
& \left.\left.+h\left[\left({ }^{(s)} \widehat{b}+{ }^{(s)} \widehat{a}\right)-u(k)\right]\right)\right)+(1 \\
& \quad-\left(h\left[\left({ }^{(s)} \widehat{Z}_{2}-{ }^{(s)} \widehat{b}+{ }^{(s)} \widehat{a}\right)-u(k)\right]\right. \\
& \left.\left.\left.+h\left[u(k)-\left(-{ }^{(s)} \widehat{b}+{ }^{(s)} \widehat{a}\right)\right]\right)\right)\right]+(1-\alpha)[(1 \\
& \quad-\left(h\left[u(k)-\left({ }^{(s)} \widehat{Z}_{1}+{ }^{(s)} \widehat{b}-{ }^{(s)} \widehat{a}\right)\right]\right.
\end{aligned}
$$




$$
\begin{aligned}
& \left.\left.+h\left[\left({ }^{(s)} \widehat{b}-{ }^{(s)} \widehat{a}\right)-u(k)\right]\right)\right)+(1 \\
& -\left(h\left[\left({ }^{(s)} \widehat{Z}_{2}-{ }^{(s)} \widehat{b}-{ }^{(s)} \widehat{a}\right)-u(k)\right]\right. \\
& \left.\left.\left.+h\left[u(k)-\left({ }^{(s)} \widehat{b}-{ }^{(s)} \widehat{a}\right)\right]\right)\right)\right], \\
& { }^{(s)} \widehat{f}_{2}(k)=\alpha\left[1-\left(h\left[u(k)-\left({ }^{(s)} \widehat{Z}_{1}+{ }^{(s)} \widehat{b}+{ }^{(s)} \widehat{a}\right)\right]\right.\right. \\
& \left.+h\left[\left({ }^{(s)} \widehat{b}+{ }^{(s)} \widehat{a}\right)-u(k)\right]\right)+(1 \\
& -\left(h\left[\left({ }^{(s)} \widehat{Z}_{2}-{ }^{(s)} \widehat{b}+{ }^{(s)} \widehat{a}\right)-u(k)\right]\right. \\
& \left.\left.\left.+h\left[u(k)-\left(-{ }^{(s)} \widehat{b}+{ }^{(s)} \widehat{a}\right)\right]\right)\right)\right]-(1-\alpha)[1 \\
& -\left(h\left[u(k)-\left({ }^{(s)} \widehat{Z}_{1}+{ }^{(s)} \widehat{b}-{ }^{(s)} \widehat{a}\right)\right]+h\left[\left({ }^{(s)} \widehat{b}-{ }^{(s)} \widehat{a}\right)\right.\right. \\
& -u(k)])+\left(1-\left(h\left[\left({ }^{(s)} \widehat{Z}_{2}-{ }^{(s)} \widehat{b}-{ }^{(s)} \widehat{a}\right)-u(k)\right]\right.\right. \\
& \left.\left.\left.+h\left[u(k)-\left(-{ }^{(s)} \widehat{b}-{ }^{(s)} \widehat{a}\right)\right]\right)\right)\right], \\
& { }^{(s)} \widehat{f}_{3}(k)=\alpha\left[\left(1-\left(h\left[u(k)-\left({ }^{(s)} \widehat{Z}_{1}+{ }^{(s)} \widehat{b}+{ }^{(s)} \widehat{a}\right)\right]\right.\right.\right. \\
& \left.\left.+h\left[\left({ }^{(s)} \widehat{b}+{ }^{(s)} \widehat{a}\right)-u(k)\right]\right)\right)-(1 \\
& -\left(h\left[\left({ }^{(s)} \widehat{Z}_{2}-{ }^{(s)} \widehat{b}+{ }^{(s)} \widehat{a}\right)-u(k)\right]\right. \\
& \left.\left.+h\left[u(k)-\left(-\left({ }^{(s)} \widehat{b}+{ }^{(s)} \widehat{a}\right)\right]\right)\right)\right]+(1-\alpha)[(1 \\
& -\left(h\left[u(k)-\left({ }^{(s)} \widehat{Z}_{1}+{ }^{(s)} \widehat{b}-{ }^{(s)} \widehat{a}\right)\right]\right. \\
& \left.\left.+h\left[\left({ }^{(s)} \widehat{b}-{ }^{(s)} \widehat{a}\right)-u(k)\right]\right)\right)-(1 \\
& -\left(h\left[\left({ }^{(s)} \widehat{Z}_{2}-{ }^{(s)} \widehat{b}-{ }^{(s)} \widehat{a}\right)-u(k)\right]\right. \\
& \left.\left.\left.+h\left[u(k)-\left(-{ }^{(s)} \widehat{b}-{ }^{(s)} \widehat{a}\right)\right]\right)\right)\right], \\
& { }^{(s)} \widehat{h}_{1}(k)=\alpha h\left[u(k)-\left({ }^{(s)} \widehat{Z}_{1}+{ }^{(s)} \widehat{b}+{ }^{(s)} \widehat{a}\right)\right]+(1-\alpha) \\
& \cdot h\left[u(k)-\left({ }^{(s)} \widehat{Z}_{1}+{ }^{(s)} \widehat{b}-{ }^{(s)} \widehat{a}\right)\right], \\
& { }^{(s)} \widehat{h}_{2}(k)=\alpha h\left[\left({ }^{(s)} \widehat{Z}_{2}-{ }^{(s)} \widehat{b}+{ }^{(s)} \widehat{a}\right)-u(k)\right]+(1-\alpha) \\
& \cdot h\left[\left({ }^{(s)} \widehat{Z}_{2}-{ }^{(s)} \widehat{b}-{ }^{(s)} \widehat{a}\right)-u(k)\right], \\
& { }^{(s)} \widehat{h}_{1 \mathrm{pr}}(k)=h\left[u(k)-\left({ }^{(s)} \widehat{b}-{ }^{(s)} \widehat{a}\right)\right], \\
& { }^{(s)} \widehat{h}_{2 \mathrm{pr}}(k)=h\left[\left(-{ }^{(s)} \widehat{b}+{ }^{(s)} \widehat{a}\right)-u(k)\right] .
\end{aligned}
$$

The proposed iterative algorithm is summarized as follows.

Step 1 (initialization). ${ }^{(0)} \widehat{g}_{\text {UDN }}^{T}$ and ${ }^{(0)} \widehat{\varphi}_{\text {UDN }}(k)$ and the stopping criteria $\lambda$.

Step 2. $s=s+1$; construct the information vector ${ }^{(s)} \widehat{\varphi}_{\mathrm{UDN}}(k)$.

Step 3. Estimate the system matrices $\left({ }^{(s)} \widehat{A},{ }^{(s)} \widehat{\widetilde{B}},{ }^{(s)} \widehat{C},{ }^{(s)} \widehat{\widetilde{D}}\right)$ and the model order $n$ using MOESP algorithm.

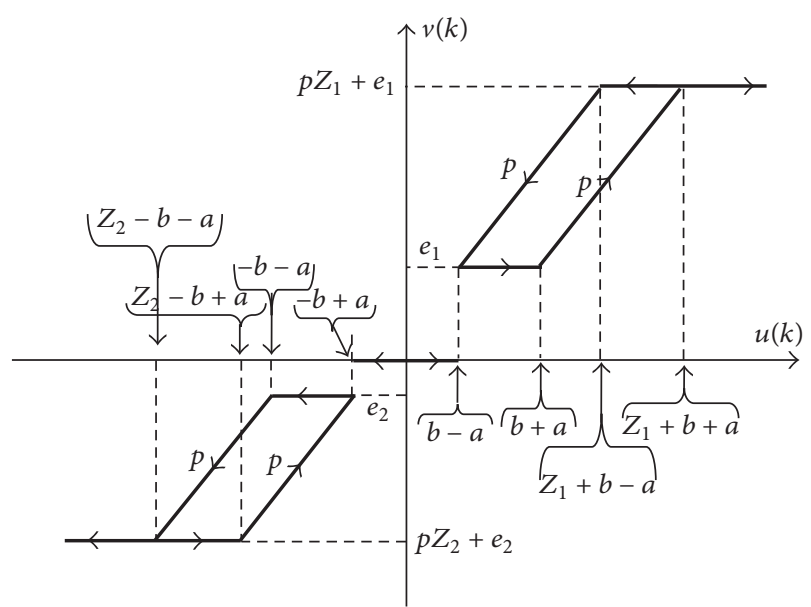

Figure 2: Unified Discontinuous Nonlinearity.

Step 4. Compute the SVD of ${ }^{(s)} \widehat{\widetilde{\Theta}}_{B D}$ using (16).

Step 5. Computes the matrices parameter ${ }^{(s)} \widehat{B},{ }^{(s)} \widehat{D}$, and ${ }^{(s)} \widehat{g}_{\text {UDN }}$ as

$$
\begin{aligned}
& {\left[\begin{array}{l}
{ }^{(s)} \widehat{B} \\
{ }^{(s)} \widehat{D}
\end{array}\right]={ }^{(s)} U_{1}{ }^{(s)} \Sigma_{1},} \\
& { }^{(s)} \widehat{g}_{\mathrm{UDN}}={ }^{(s)} V_{1} \text {, } \\
& { }^{(s)} \widehat{\Theta}=\left[\begin{array}{ll}
{ }^{(s)} & { }^{(s)} \widehat{B} \\
{ }^{(s)} & { }^{(s)} \widehat{D}
\end{array}\right] .
\end{aligned}
$$

Step 6. Calculate Mean Square Error (MSE) using

$$
{ }^{(s)} \mathrm{MSE}=\frac{1}{N} \sum_{k=1}^{N}\left(y(k)-{ }^{(s)} \hat{y}(k)\right)^{2},
$$

with

$$
\begin{aligned}
{ }^{(s)} \widehat{x}(k+1) & ={ }^{(s)} \widehat{A}^{(s)} \hat{x}(k)+{ }^{(s)} \widehat{\tilde{B}}^{(s)} \widehat{\varphi}_{\text {new }}(k), \\
{ }^{(s)} \hat{y}(k) & ={ }^{(s)} \widehat{C}^{(s)} \widehat{x}(k)+{ }^{(s)} \widehat{\widetilde{D}}^{(s)} \widehat{\varphi}_{\text {new }}(k),
\end{aligned}
$$

where $N$ is the number of data $y_{k}$ and ${ }^{(s)} \hat{y}_{k}$ denotes the real and the estimated outputs.

If $\left\|{ }^{(s)} \mathrm{MSE}-{ }^{(s-1)} \mathrm{MSE}\right\|<\lambda$, then $\widehat{\Theta}={ }^{(s)} \widehat{\Theta}$ and $\widehat{g}_{\text {UDN }}=$ ${ }^{(s)} \widehat{g}_{\text {UDN }}$. Otherwise, return to Step 2.

\section{Result and Discussions}

In this section, we are going to examine the performance of the proposed method developed above.

In the first step, we consider the unified form of the nonlinearity to determine the structure of the nonlinearity of the system. Once the nonlinearity is recognized, in the second step, we use it to identify the model with the exact form of the nonlinearity. 


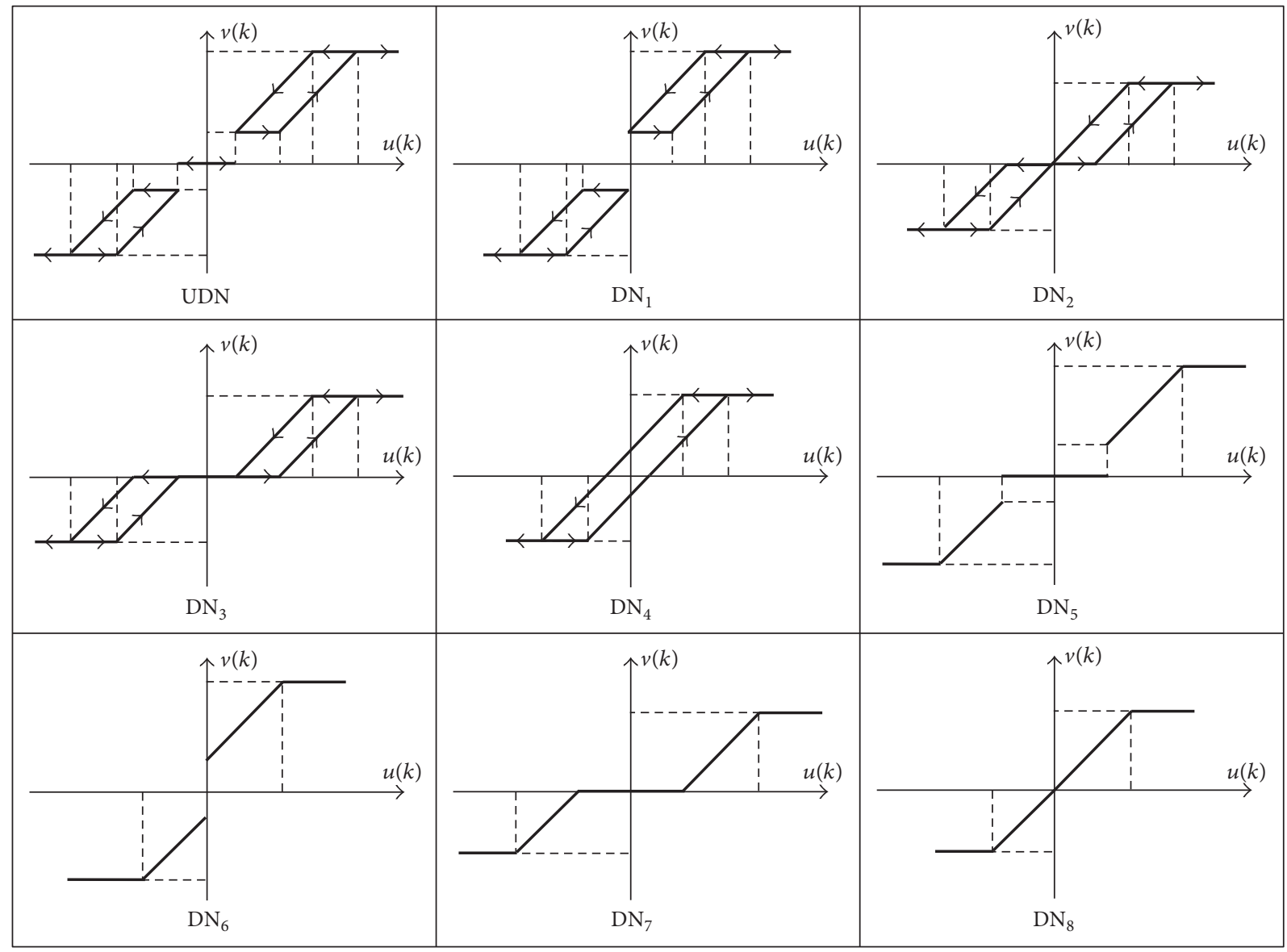

FiguRE 3: Different cases of nonlinearities.

4.1. Example 1. We considered Hammerstein model where the parameters characterizing the $\mathrm{DN}_{4}$ nonlinearity are $p=$ 2, $a=0.5, b=0, Z_{1}=1.5, Z_{2}=-1, e_{1}=0$, and $e_{2}=0$.

The LTI state space model $S(\cdot)$ can be determined by looking at the system transfer function in factored form

$$
G(z)=\frac{z^{-1}\left(b_{1}+b_{2} z^{-1}\right)}{1+a_{1} z^{-1}+a_{2} z^{-2}}=\frac{z^{-1}\left(1+0.5 z^{-1}\right)}{1-0.2 z^{-1}+0.35 z^{-2}} .
$$

The iterative identification procedure was carried out for 2000 samples of uniformly distributed random inputs with $|u(k)| \leq$ 3. $\varepsilon(k)$ is a white noise with zero mean and variance $\sigma^{2}$, which is added to the output system at different SNR levels (SNR = 10 and $8 \mathrm{~dB}$ ), in order to demonstrate the effectiveness of the proposed algorithm in a noisy environment.

The Monte Carlo experiments are carried out with 50 runs. The average estimated value $\left(\overline{l_{i}}\right)$ and the standard deviation value $\left(\sigma_{l_{i}}\right)$ obtained in the noisy case are

$$
\begin{aligned}
\bar{l}_{i} & =\frac{1}{M} \sum_{m=1}^{M} l_{i, m}, \\
\sigma_{l_{i}} & =\sqrt{\frac{1}{M} \sum_{m=1}^{M}\left(l_{i, m}-\bar{l}_{i}\right)^{2}},
\end{aligned}
$$

where $M$ is the number of replication and $\widehat{l}_{i}=\bar{l}_{i} \pm \sigma_{l_{i}}$.
The structure of the nonlinearity is assumed unknown. For this reason, we begin with the first step to find the type of the nonlinearity using the UDN.

The true parameters and the estimated parameters of nonlinear and linear blocks for the Hammerstein model are listed in Table 2. Figure 4 represents the nonlinearity estimated by model UDN for different values of SNR.

From Figure 4, we can observe that the nonlinearity of this system corresponds to a saturation with hysteresis $\left(\mathrm{DN}_{4}\right)$ in the absence of noise. As shown in Table 2 and Figure 4, the proposed method present good performance for the lower level of noise. With higher level of noise ( $\mathrm{SNR}=8 \mathrm{~dB}$ ), the proposed algorithms exhibit poor performance. Despite that, we were able to identify the model structure of nonlinear block. Once the structure of nonlinear block is determined, we will resume again the identification steps using the exact model.

We considered the parameters characterizing the $\mathrm{SH}$ nonlinearity: $a=0.5, p=2, Z_{1}=1.5$, and $Z_{2}=-1$. The true and the estimated parameters of nonlinear and linear blocks for the Hammerstein model with SH nonlinearity are summarized in Table 3.

The comparison simulation results with $\mathrm{SH}$ nonlinearity are given in Table 3 with different SNR levels.

As seen in Table 3, our method with the parameters of the exact structure of nonlinear block gives the best MSE 
TABLE 2: Parameters estimation for the Hammerstein model with SNR $=10$ and $8 \mathrm{~dB}$.

\begin{tabular}{|c|c|c|c|c|c|c|c|c|c|c|c|c|c|}
\hline \multirow{2}{*}{ SNR } & \multicolumn{6}{|c|}{ Parameters for the nonlinear model } & \multicolumn{4}{|c|}{ Denominator } & \multicolumn{2}{|c|}{ Numerator } & \multirow{2}{*}{ MSE } \\
\hline & & $p$ & $a$ & $b$ & $Z_{1}$ & $Z_{2}$ & $e_{1}$ & $e_{2}$ & $a_{1}$ & $a_{2}$ & $b_{1}$ & $b_{2}$ & \\
\hline \multirow{3}{*}{$\begin{array}{l}\text { Without } \\
\text { noise }\end{array}$} & True & 2.0000 & 0.5000 & 0.0000 & 1.5000 & -1.0000 & 0.0000 & 0.0000 & -0.2000 & 0.3500 & 1.0000 & 0.5000 & \multirow{3}{*}{0.0004} \\
\hline & $\bar{l}_{i}$ & 2.0000 & 0.5001 & 0.0000 & 1.5001 & -1.0000 & 0.0006 & 0.0007 & -0.2001 & 0.3500 & 1.0013 & 0.4998 & \\
\hline & $\sigma_{l_{i}}$ & 0.0001 & 0.0003 & 0.0001 & 0.0005 & 0.0000 & 0.0004 & 0.0007 & 0.0001 & 0.0000 & 0.0002 & 0.0002 & \\
\hline \multirow{3}{*}{$10 \mathrm{~dB}$} & True & 2.0000 & 0.5000 & 0.0000 & 1.5000 & -1.0000 & 0.0000 & 0.0000 & -0.2000 & 0.3500 & 1.0000 & 0.5000 & \multirow{3}{*}{0.0437} \\
\hline & $\overline{l_{i}}$ & 2.0027 & 0.4760 & 0.0326 & 1.4874 & -1.0546 & 0.0538 & -0.0368 & -0.2006 & 0.3496 & 1.0029 & 0.4898 & \\
\hline & $\sigma_{l_{i}}$ & 0.0709 & 0.0618 & 0.0208 & 0.0264 & 0.0188 & -0.0573 & 0.0189 & 0.0017 & 0.0010 & 0.0091 & 0.0073 & \\
\hline \multirow{3}{*}{$8 \mathrm{~dB}$} & True & 2.0000 & 0.5000 & 0.0000 & 1.5000 & -1.0000 & 0.0000 & 0.0000 & -0.2000 & 0.3500 & 1.0000 & 0.5000 & \multirow{3}{*}{0.1596} \\
\hline & $\bar{l}_{i}$ & 2.0741 & 0.5341 & 0.1018 & 1.4210 & -0.8515 & -0.0715 & -0.0290 & -0.1980 & 0.3488 & 1.0385 & 0.4887 & \\
\hline & $\sigma_{l_{i}}$ & 0.0388 & 0.0106 & 0.0287 & 0.0941 & 0.0963 & 0.1068 & 0.0327 & 0.0016 & 0.0022 & 0.0247 & 0.0783 & \\
\hline
\end{tabular}

TABLE 3: Parameters estimation for the Hammerstein model with $\mathrm{SNR}=10,8$, and $5 \mathrm{~dB}$.

\begin{tabular}{|c|c|c|c|c|c|c|c|c|c|c|}
\hline \multirow{2}{*}{ SNR } & & \multicolumn{4}{|c|}{ Parameters for the nonlinear model } & \multicolumn{2}{|c|}{ Denominator } & \multicolumn{2}{|c|}{ Numerator } & \multirow{2}{*}{ MSE } \\
\hline & & $p$ & $a$ & $Z_{1}$ & $Z_{2}$ & $a_{1}$ & $a_{2}$ & $b_{0}$ & $b_{1}$ & \\
\hline \multirow{3}{*}{ Without noise } & True & 2.0000 & 0.5000 & 1.5000 & -1.0000 & -0.2000 & 0.3500 & 1.0000 & 0.5000 & \multirow{3}{*}{$4.10^{-6}$} \\
\hline & $\bar{l}_{i}$ & 2.0000 & 0.5000 & 1.5000 & -1.0000 & -0.2000 & 0.3500 & 0.9998 & 0.5001 & \\
\hline & $\sigma_{l_{i}}$ & 0.0001 & 0.0001 & 0.0001 & 0.0001 & 0.0001 & 0.0000 & 0.0001 & 0.0001 & \\
\hline \multirow{3}{*}{$10 \mathrm{~dB}$} & True & 2.0000 & 0.5000 & 1.5000 & -1.0000 & -0.2000 & 0.3500 & 1.0000 & 0.5000 & \multirow{3}{*}{0.0129} \\
\hline & $\overline{l_{i}}$ & 2.0125 & 0.4955 & 1.4884 & -0.9971 & -0.2039 & 0.3468 & 0.9844 & 0.4939 & \\
\hline & $\sigma_{l_{i}}$ & 0.0149 & 0.0169 & 0.0154 & 0.0218 & 0.0067 & 0.0041 & 0.0248 & 0.0149 & \\
\hline \multirow{3}{*}{$8 \mathrm{~dB}$} & True & 2.0000 & 0.5000 & 1.5000 & -1.0000 & -0.2000 & 0.3500 & 1.0000 & 0.5000 & \multirow{3}{*}{0.0263} \\
\hline & $\overline{l_{i}}$ & 1.9881 & 0.4925 & 1.4877 & -0.9964 & -0.1964 & 0.3455 & 1.0198 & 0.5081 & \\
\hline & $\sigma_{l_{i}}$ & 0.0127 & 0.0304 & 0.0275 & 0.0278 & 0.0246 & 0.0213 & 0.0354 & 0.0358 & \\
\hline \multirow{3}{*}{$5 \mathrm{~dB}$} & True & 2.0000 & 0.5000 & 1.5000 & -1.0000 & -0.2000 & 0.3500 & 1.0000 & 0.5000 & \multirow{3}{*}{0.0396} \\
\hline & $\overline{l_{i}}$ & 2.0776 & 0.4824 & 1.4724 & -0.9270 & -0.2232 & 0.3328 & 0.9686 & 0.5198 & \\
\hline & $\sigma_{l_{i}}$ & 0.0144 & 0.0731 & 0.0308 & 0.0205 & 0.0455 & 0.0458 & 0.0153 & 0.0215 & \\
\hline
\end{tabular}

performance without and with noise. A similar analysis can be seen also in Figure 5.

4.2. Example 2: Electropneumatic System. We use the proposed method to identify the parameters of an industrial process. The process is an electropneumatic system and shown in Figure 6 . The actual system consists of a carriage, a proportional valve $5 / 3$, and a linear position sensor (linear potentiometer).

The proportional distributor 5/3, shown in Figure 7, converts the analog electrical input signal into the openings of the cross sections adequate to the outputs. In midnominal voltage $(5 \mathrm{~V})$, the distributor takes its pneumatic position in which all the control edges are closed. In both directions (ascending and descending), the distributor witnesses a voltage-variation and this leads to the emergence of a hysteresis phenomenon.

The linear potentiometer is used to measure the movement of the carriage. The latter converts the linear position

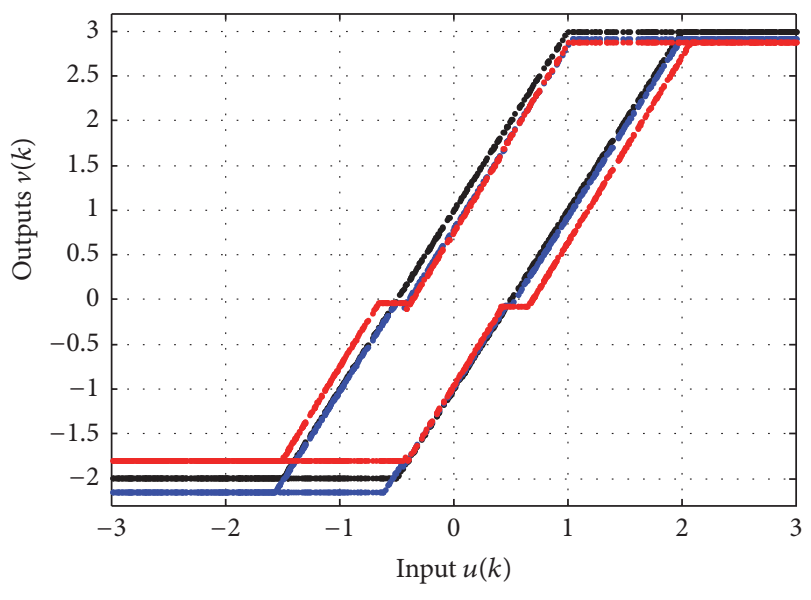

- $\mathrm{DN}_{4}$ estimated without noise

- $\mathrm{DN}_{4}$ estimated with $\mathrm{SNR}=10 \mathrm{~dB}$

- $\mathrm{DN}_{4}$ estimated with $\mathrm{SNR}=8 \mathrm{~dB}$

FIGURE 4: $\mathrm{DN}_{4}$ estimated with UDN model. 
TABLE 4: Estimates of the parameters for the nonlinear model.

\begin{tabular}{lccccccr}
\hline \multirow{2}{*}{ Nonlinearity } & \multicolumn{9}{c}{ Parameters nonlinear block } & \multicolumn{2}{c}{ MSE } \\
& $p$ & $a$ & $b$ & $Z_{1}$ & $Z_{2}$ & $e_{1}$ & $e_{2}$ \\
\hline UDN & 6.2850 & 0.6536 & 0.0701 & 0.5136 & -0.5058 & 0.2785 & -0.2512 \\
SH & 8.9668 & 0.5740 & - & 0.4016 & -0.3973 & - & - \\
\hline
\end{tabular}

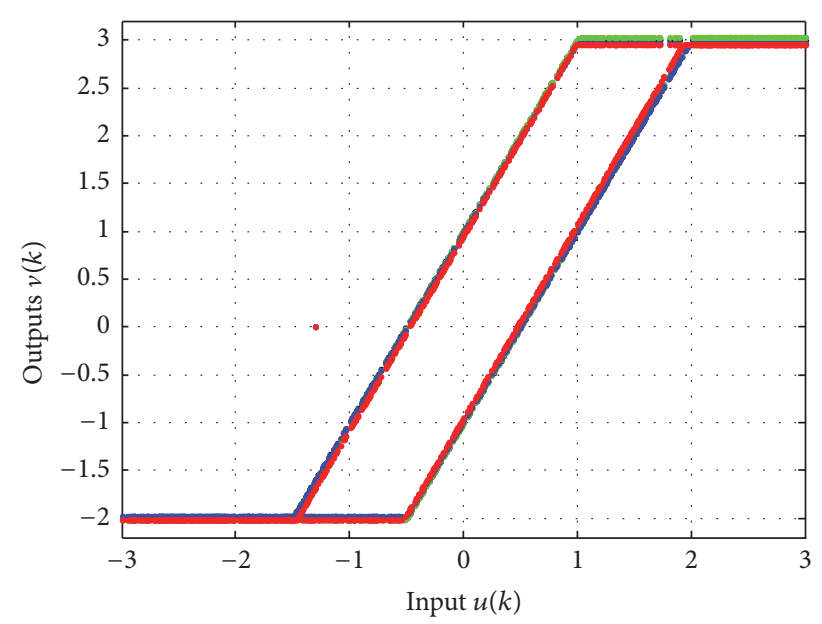

- NL SH estimated without noise

- NL SH estimated with SNR = $10 \mathrm{~dB}$

- NL SH estimated with $\mathrm{SNR}=8 \mathrm{~dB}$

- NL SH estimated with SNR $=5 \mathrm{~dB}$

FIGURE 5: Nonlinearity SH estimated without SNR and with SNR = 10,8 et $5 \mathrm{~dB}$.

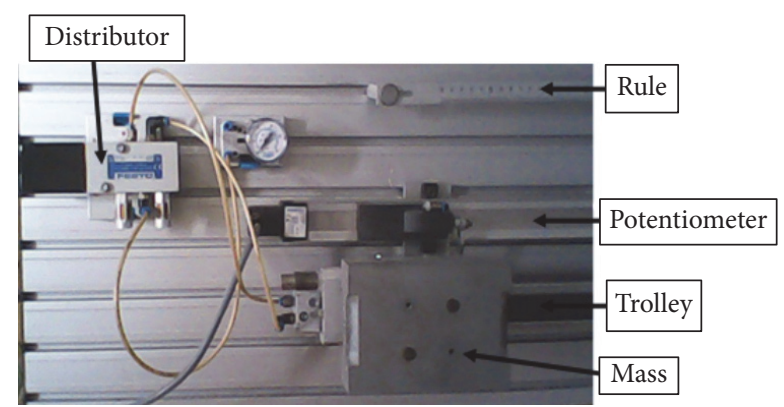

FIGURE 6: Electropneumatic system.

into an output voltage which is proportional to that of the supply and the cursor position. This is performed by using the voltage divider principle.

Figure 8 shows the static characteristic of the proportional distributor given by the manufacturer corresponding to a single orifice 4 . A distributor test is achieved by varying the voltage in both directions (ascending and descending) with opening orifice 4 .

We try to structurally identify the discontinuous static nonlinearity in the first step. To do this, we use the model of Hammerstein based on the UDN model. Two sets of inputs/ outputs datasets (Figures 9 and 10) are used to the system identification and validation. For each set, the first one is used as training data and the second one is used as test data.

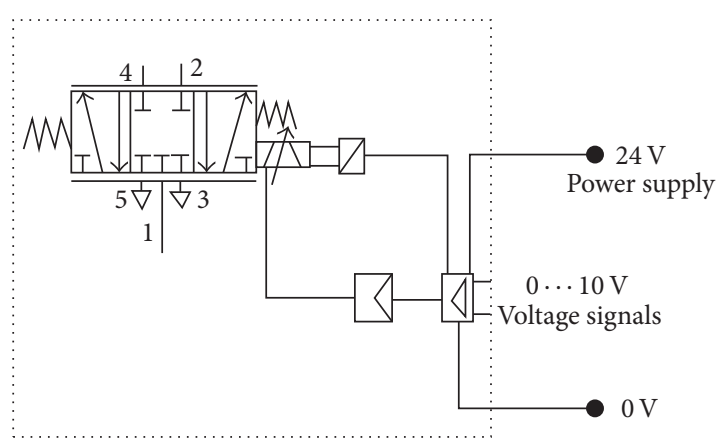

FIGURE 7: Distributor connection scheme.

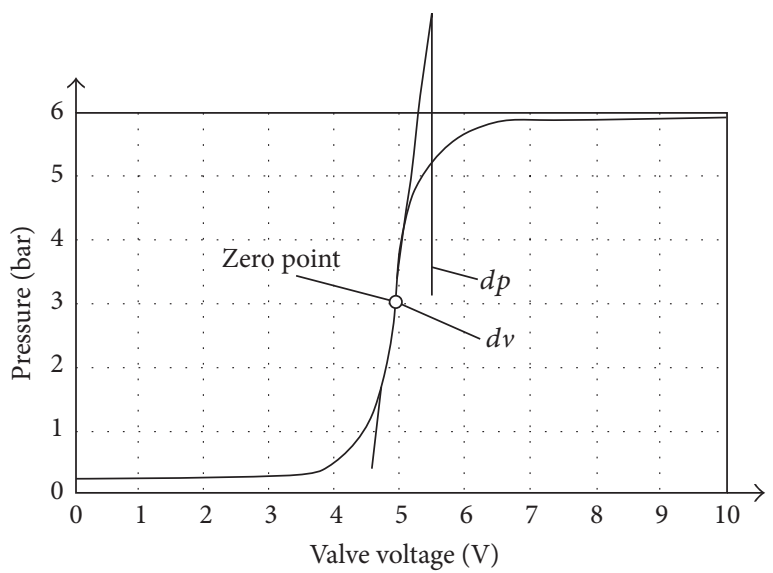

FIGURE 8: Manufacturer test of static characteristic of the proportional distributor $5 / 3$.

The identified parameters obtained by our method are listed in Table 4. This table shows the average estimated value $\left(\overline{l_{i}}\right)$ for the UDN and SH nonlinearities.

Through Figure 11, we can conclude that the UDN corresponds to the structure of $\mathrm{DN}_{4}$ regarding Figure 3. As shown in Figure 11, the $\mathrm{DN}_{4}$ gives a good characterization of the nonlinearity (the hysteresis phenomenon of the distributor 5/3) for the electropneumatic system. The MSEs of the estimates of the model using UDN is equal to 0.0475 and using $\mathrm{SH}$ is 0.0366 when the Hammerstein is taken with an exact nonlinearity.

Using the second datasets (Figures 9 and 10) for system validation, the performance of the proposed method is shown in Figure 12. We notice that the identification quality improves, especially at the level of nonlinearity. From Figure 12, it can be seen that our method provides a good approximation modelling accuracy. 


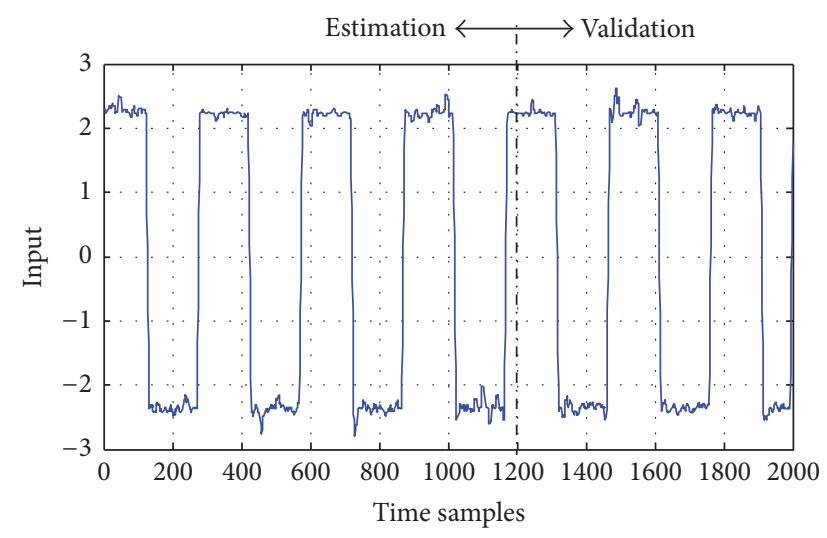

FIGURE 9: First set of measurement input after treatment.

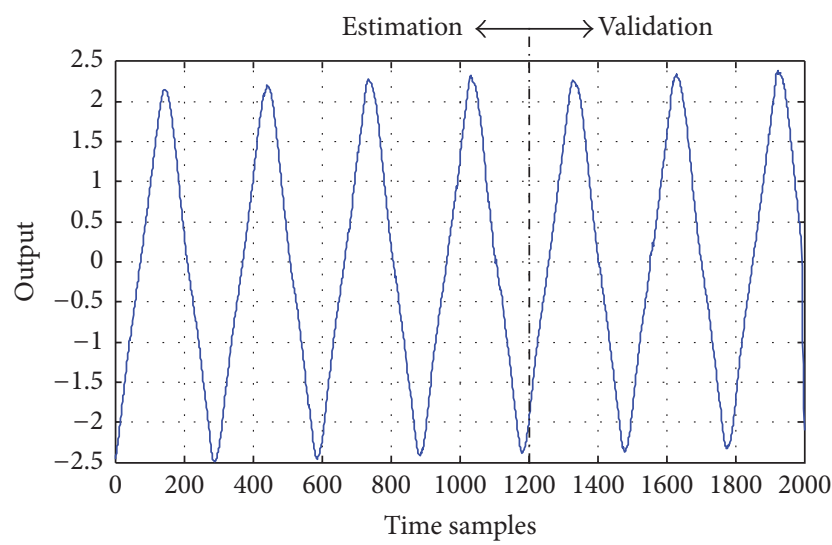

FIGURE 10: First set of measurement output after treatment.

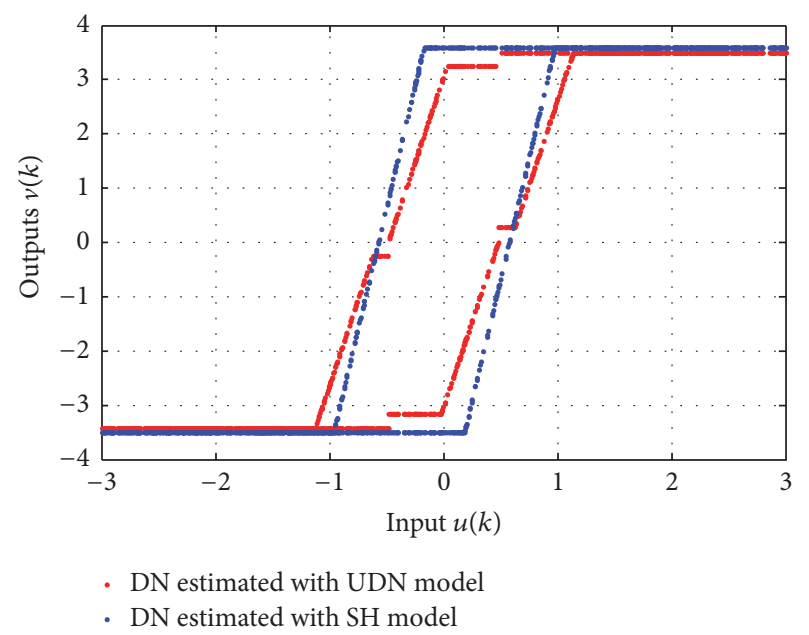

FIGURE 11: Nonlinearity with model UDN and SH structures.

\section{Conclusion}

In this paper, a new iterative subspace algorithm for the Hammerstein model identification is presented. The application of the proposed method improves a major advantage which is the simultaneous identification of the parameters of the linear

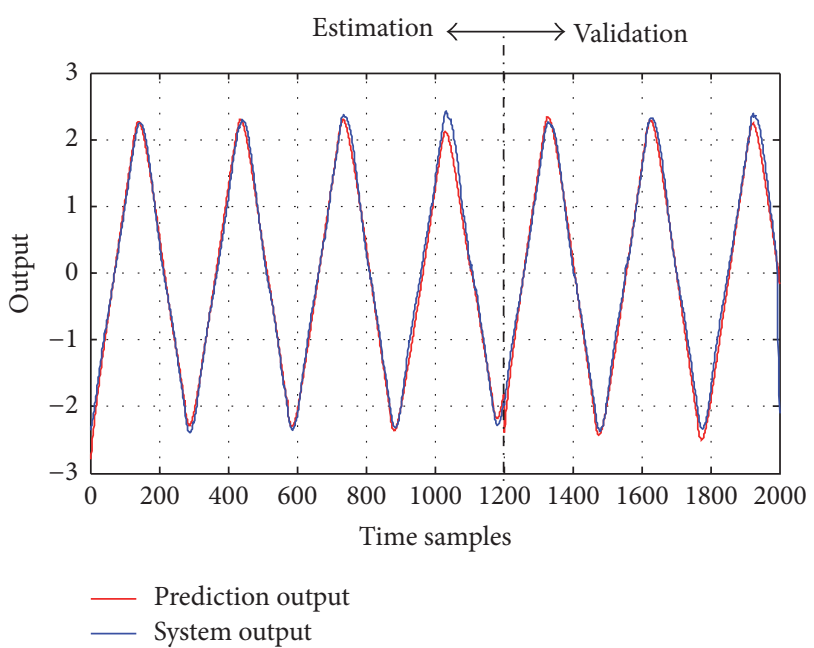

FIGURE 12: The real and the estimated outputs for the training and testing data sets.

and nonlinear blocks. Indeed, these two blocks are assumed unknown. The use of UDN model with Hammerstein model can be identified the adapted structure for a given system with and without noise. Furthermore, an iterative subspace algorithm is used to finally tune the parameters of model.

Two examples are presented to verify the effectiveness of the proposed approach. As a result, the proposed method is reliable to describe approximately the real nonlinearity of such as systems and can be applied to other various industrial systems to a level of well-defined noise.

\section{Competing Interests}

The authors declare that they have no competing interests.

\section{References}

[1] K. Jalaleddini and R. E. Kearney, "Subspace identification of SISO hammerstein systems: application to stretch reflex identification," IEEE Transactions on Biomedical Engineering, vol. 60, no. 10, pp. 2725-2734, 2013.

[2] G. N. Maksym, R. E. Kearney, and J. H. T. Bates, "Nonparametric block-structured modeling of lung tissue strip mechanics," Annals of Biomedical Engineering, vol. 26, no. 2, pp. 242-252, 1998.

[3] K. S. Narendra and P. G. Gallman, "An iterative method for the identification of nonlinear systems using a Hammerstein model," IEEE Transactions on Automatic Control, vol. 11, no. 3, pp. 546-550, 1966.

[4] I. W. Hunter and M. J. Korenberg, "The identification of nonlinear biological systems: Wiener and Hammerstein cascade models," Biological Cybernetics, vol. 55, no. 2-3, pp. 135-144, 1986.

[5] J. Chen and X. Wang, "Identification of Hammerstein systems with continuous nonlinearity," Information Processing Letters, vol. 115, no. 11, pp. 822-827, 2015.

[6] A. Chaari, S. Rejeb, F. B. Hmida, H. Messaoud, and M. Gossa, "Iterative identification of Wiener model using hysteresis memory-less nonlinearity," International Journal of Sciences and 
Techniques of Automatic Control \& Computer Engineering, vol. 2, no. 1, pp. 430-441, 2008.

[7] F. Giri, Y. Rochdi, F. Z. Chaoui, and A. Brouri, "Identification of Hammerstein systems in presence of hysteresis-backlash and hysteresis-relay nonlinearities," Automatica, vol. 44, no. 3, pp. 767-775, 2008.

[8] J. Vörös, "Identification of nonlinear cascade systems with timevarying backlash," Journal of Electrical Engineering, vol. 62, no. 2, pp. 87-92, 2011.

[9] S. Rejeb, R. B. H. Ali, F. B. Hmida, A. Chaari, and H. Messaoud, "Recursive identification of Hammerstein ARMAX model with general discontinuous nonlinearity," Scientific Research and Essays, vol. 6, no. 23, pp. 4925-4935, 2011.

[10] S. Rejeb, F. B. Hmida, A. Chaari, and M. Gossa, "Design and parameter identification of a general Hammerstein model," in Proceedings of the 8th IEEE International Multi-Conference on Systems, Signals \& Devices (SSD '11), March 2011.

[11] J. Vörös, "Parametric identification of systems with general backlash," Informatica, vol. 23, no. 2, pp. 283-298, 2012.

[12] D. Westwick and R. Kearney, Identification of Nonlinear Physiological Systems, John Wiley \& Sons, New York, NY, USA, 2003.

[13] M. Verhaegen and P. Dewilde, "Subspace model identification part 1 . The output-error state-space model identification class of algorithms," International Journal of Control, vol. 56, no. 5, pp. 1187-1210, 1992.

[14] I. Goethals, K. Pelckmans, J. A. K. Suykens, and B. De Moor, "Identification of MIMO Hammerstein models using least squares support vector machines," Automatica, vol. 41, no. 7, pp. 1263-1272, 2005.

[15] M. Verhaegen and A. Varga, "Some experience with the MOESP class of subspace model identification methods in identifying the BO105 helicopter," Tech. Rep., German Aerospace Research Establishment, Laboratory for Flight Systems Dynamics, Wessling, Germany, 1994.

[16] M. Verhaegen and D. Westwick, "Identifying MIMO Hammerstein systems in the context of subspace model identification methods," International Journal of Control, vol. 63, no. 2, pp. 331349, 1996.

[17] J. C. Gomez and E. Baeyens, "Subspace-based identification algorithms for Hammerstein and Wiener models," European Journal of Control, vol. 11, no. 2, pp. 127-136, 2005.

[18] S. Marchesiello and L. Garibaldi, "Subspace-based identification of nonlinear structures," Shock and Vibration, vol. 15, no. 3-4, pp. 345-354, 2008.

[19] E. W. Bai, "Discussion on: subspace-based identification algorithms for Hammerstein and Wiener models," European Journal of Control, vol. 11, no. 2, pp. 137-138, 2005.

[20] H. Ase and T. Katayama, "A subspace-based identification of Wiener-Hammerstein benchmark model," Control Engineering Practice, vol. 44, pp. 126-137, 2015.

[21] D. N. Miller and R. A. de Callafon, "Subspace identification with eigenvalue constraints," Automatica, vol. 49, no. 8, pp. 24682473, 2013.

[22] Y. Li, C. Liao, L. Wang, L. Wang, and D. Xu, "Subspace-based modeling and parameter identification of lithium-ion batteries," International Journal of Energy Research, vol. 38, no. 8, pp. 10241038, 2014.

[23] C. Yu and M. Verhaegen, "Blind multivariable ARMA subspace identification," Automatica, vol. 66, pp. 3-14, 2016.

[24] P. Van Overschee and B. De Moor, "A unifying theorem for three subspace system identification algorithms," Automatica, vol. 31, no. 12, pp. 1853-1864, 1995.
[25] G. H. Golub and C. F. Van Loan, Matrix Computations, The Johns Hopkins University Press, Baltimore, Md, USA, 2nd edition, 1989. 


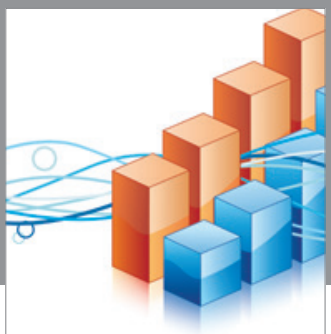

Advances in

Operations Research

vatem alat4

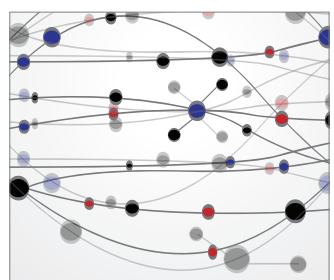

\section{The Scientific} World Journal
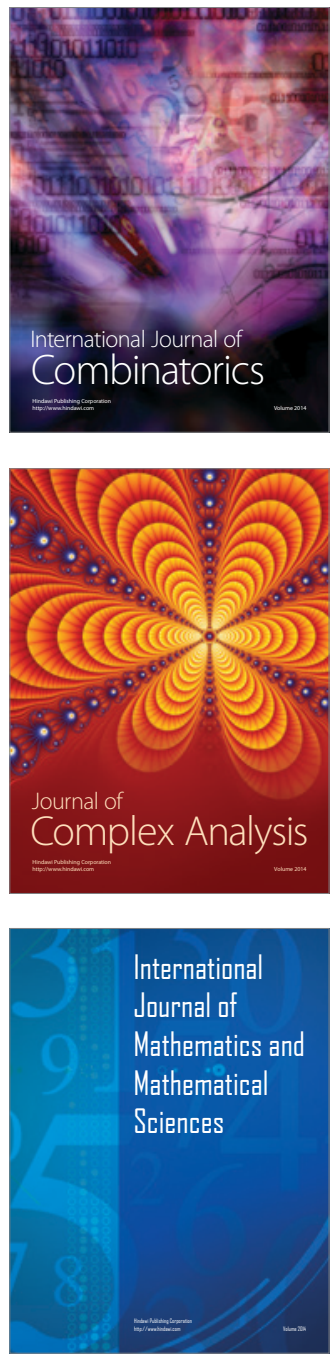
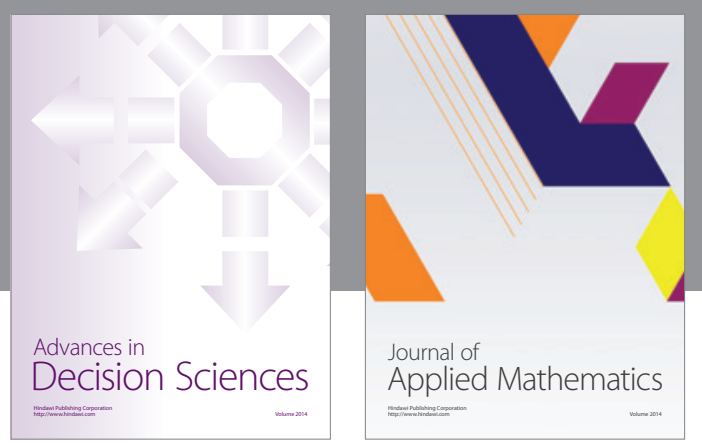

Algebra

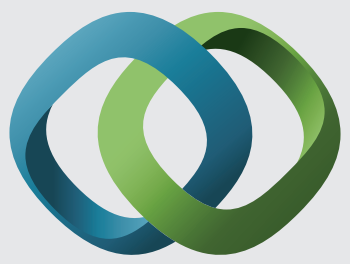

\section{Hindawi}

Submit your manuscripts at

http://www.hindawi.com
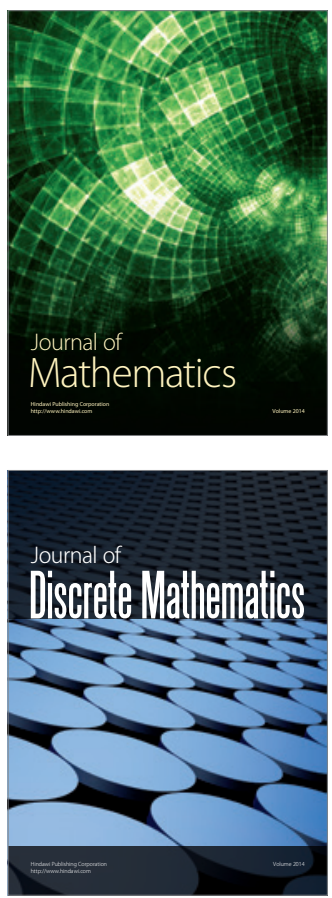

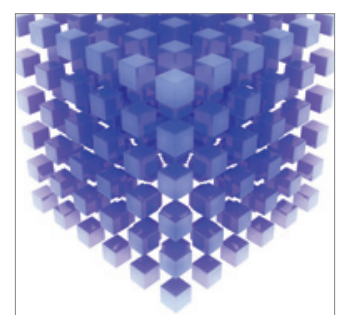

Mathematical Problems in Engineering
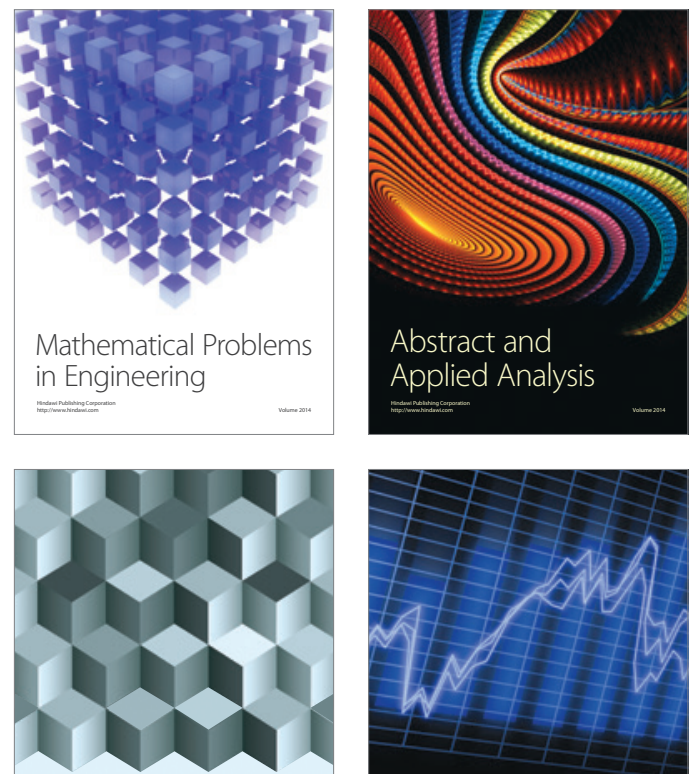

Journal of

Function Spaces

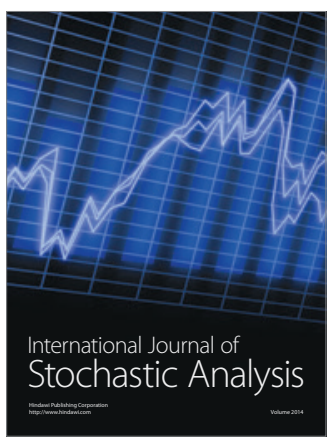

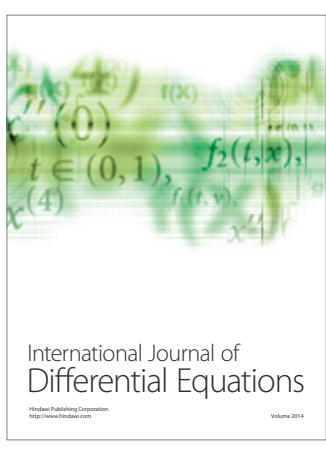
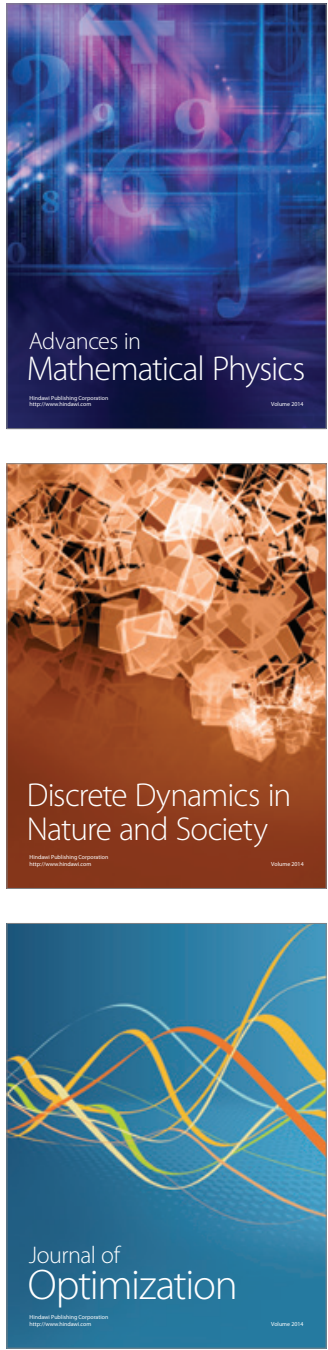